RESEARCH NOTE

\title{
A theoretical investigation of band gaps of conducting polymers with heterocycles
}

\author{
M.S. Senevirathne, A. Nanayakkara ${ }^{*}$ and G.K.R. Senadeera \\ Institute of Fundamental Studies, Hanthana Road, Kandy.
}

Revised: 02 May 2011 ; Accepted: 17 December 2010

Keywords: Conducting polymers, DFT calculations, polyfurane, polypyrrole, polythiophene.

This study reports a new method developed for calculating band gaps of conducting polymers. The method consists of three major steps. In the first step, the geometry optimization of the oligomer structures was carried out. The optimized oligomer structures were then used as input in the second step. In the second step band gaps of oligomers were calculated by taking into account the energy difference of the highest occupied molecular orbital (HOMO) and lowest unoccupied molecular orbital (LUMO) levels. In the third step, the nearly free electron model and the extrapolation techniques on the oligomers were used to obtain the band gap values of polymers.

Geometry optimization was performed on isolated oligomers of polypyrrole, polythiophene, polyfuran and some of their derivatives, using density functional theory (DFT) methods with Becke exchange (Becke, 1988) and Lee-Yang-Parr correlation (Lee et al., 1988) methods within the local-density approximation (LDA). Several geometry optimization calculations were carried out with various basis sets such as STO-3G, 6-31G, 6-31G*, $6-31 \mathrm{G}^{* *}$ and $6-311 \mathrm{G}$ to figure out the optimal basis set. For calculations on the conjugated organic oligomers, which are studied here, the best basis set was found to be $6-31 \mathrm{G}^{* *}$ and was used in both geometry optimizations and band gap calculations. 6-31G** was large enough to give fairly good results while small enough to be used with a computer with 1 gigabyte random access memory (RAM). The energies corresponding to the HOMO and LUMO were extracted from the final equilibrium geometries of each oligomer. Thereafter, $\Delta E$, which is the energy difference between the HOMO and LUMO, was calculated for each oligomer.
To obtain the electronic band gaps of polymers, it is necessary to extrapolate results obtained for finite length oligomers to infinite length oligomer chains. Conjugated one-dimensional $\pi$ systems such as the oligomers used in this study are often modelled by a free electron model. But a free electron model does not recover finite band gaps at infinite lengths. Hans Kuhn (1949) introduced a more realistic method by replacing free electron model with a sine shaped potential along the chain. Assuming that the easily displaceable $\pi$ electrons are moving along the zigzag chain of single and double bond pairs and that they are in a one dimensional potential field, which has a sine wave variation along the chain, Kuhn's result (Mintmire, 1988) can be obtained as,

$$
\Delta E=\frac{h^{2}}{8 m L^{2}}(N+1)+V_{0}\left(1-\frac{1}{N}\right)
$$

Where, $h$ is the Plank's constant, $m$ is the mass of the electron, $N$ is the number of $\pi$ electrons, $L$ is the chain length (or the extension of the $\pi$ conjugated system) and $V_{0}$ is the amplitude of the sine wave variation of $\pi$ electrons. The chain length $L$ is more precisely given by, $L=(N+l) d$, where $d$ is the averaged C-C and $\mathrm{C}=\mathrm{C}$ bond length and $l$ is the effective length of the conjugated $\pi$ system (i.e. $l$ is the total number of double bond-single bond pairs along the chain).

The $V_{0}$ can be considered as the band gap of the corresponding infinitely long chain of the isolated oligomer. Now equation (1) can be written in the following form.

$V_{0}(n)=N(n)\left\{\Delta E-\frac{h^{2}}{8 m L^{2}}(N(n)+1)\right\} /(N(n)-1)$ 
where $n$ is the number of monomer units. For each oligomer, by using equation (2), $V_{0}$ is calculated from the HOMO - LUMO band gap $\Delta E$, which was obtained by DFT methods as described before. In other words, $V_{0}$ represents the band gap of the infinite chain, which produces correct $\Delta E$ of the finite oligomer. Then by plotting $V_{0}(n)$ vs $1 / n$ and extrapolating $V_{0}(n)$, the value for $V_{0}(\infty)$ is obtained. This $V_{0}(\infty)$ is now corresponding to the band gap of the polymer.

First geometry optimization calculations were carried out on oligomers of thiophene, pyrrole, furan, 3-methyl thiophene, 3-methyl pyrrole, and 3-methyl furan and then the band gaps of the corresponding polymers were calculated by extrapolation. Becke exchange and LeeYang-Parr correlation methods (Becke, 1988; Lee et al., 1988) were used for all the DFT calculations.

The estimated values of $V_{0}$ using equation (2) corresponding to oligomers of each polymer were plotted against the reciprocal of the number of monomer units (Figure 1). By finding the value of $V_{0}$ where each line intersects the $y$-axis in Figure $1, V_{0}(\infty)$ or the band gaps of the corresponding polymers were estimated. Table 1 shows the calculated values based on this method along with the previously reported experimental values in the literature.

Table 1: Theoretically calculated band gaps of conducting polymers with heterocycles

\begin{tabular}{|c|c|c|c|c|}
\hline Molecule & $\mathrm{V}_{\mathrm{o}}(\mathrm{eV})$ & $\begin{array}{c}\mathrm{V}_{\mathrm{o}}(\text { Corrected }) \\
(\mathrm{eV})\end{array}$ & $\begin{array}{c}\text { Experimental } \\
(\mathrm{eV})\end{array}$ & Reference \\
\hline Polythiophene & 0.75 & 1.8 & $1.8-2.21$ & $\begin{array}{l}\text { Mintmire et al., } 1988 \text { and } \\
\text { Kaeriyama et al., } 1989\end{array}$ \\
\hline Polypyrrole & 1.4 & 3.3 & $2.9-3.2$ & $\begin{array}{l}\text { Mintmire et al., } 1988 \text { and } \\
\text { Zotti et al., } 1992\end{array}$ \\
\hline Polyfuran & 1.05 & 2.5 & $1.94-2.7$ & $\begin{array}{l}\text { Mintmire et al., } 1988 \text { and } \\
\text { Breeze } \text { et al., } 2001\end{array}$ \\
\hline Poly 3-methyl thiophene & 0.71 & 1.7 & $1.7-2.4$ & Nguyen-Cong et al., 1993 \\
\hline Poly 3-methyl pyrrole & 1.23 & 2.9 & 2.93 & Kaeriyama et al., 1989 \\
\hline Poly 3-methyl furan & 0.90 & 2.1 & & \\
\hline
\end{tabular}

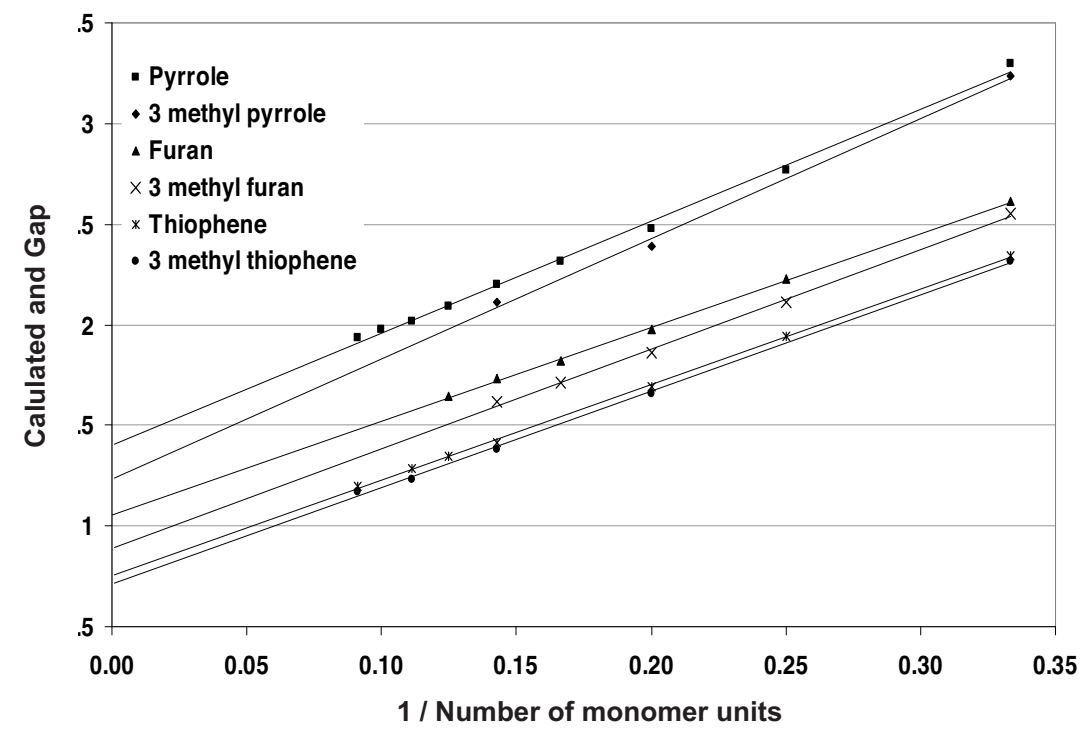

Figure 1: Plot of the calculated band gaps of oligomers against the reciprocal of corresponding oligomer lengths, i.e. the number of monomer units 
Although there is a discrepancy between experimental and theoretical values, experimental trend is correctly predicted by the theoretical calculations despite the fact that the complicated nature of the potential under which electrons move along the chain was simplified to a simple sine potential and the effects of disorder and three dimensional interactions of polymer chains were totally ignored. On the other hand, it is evident from Table1 that by ignoring above mentioned and other effects, theoretical values of the band gaps are nearly $42 \%$ smaller than the experimental values.

It is known that although the density functional methods accurately determine equilibrium geometries of polymers, they underestimate HOMO-LUMO gaps. Therefore, the above discrepancy is expected. However, in this study it was found that the discrepancy can be corrected with a single parameter. This single parameter is calculated by fitting with theoretically calculated band gaps with experimental band gaps of polythiophene, polypyrrole and polyfuran. Then the band gaps of poly 3-methyl thiophene, poly 3-methyl pyrrole, and poly 3-methyl furan were estimated by the correction parameter. The first two are in fairly good agreement with experimental values. The predicted band gap value for poly 3-methyl furan cannot be tested as experimental band gap values for poly 3-methyl furan are not available in the literature.

\section{References}

1. Breeze A. J., Schlesinger Z. \& Carter S. A. (2001). Charge transport in $\mathrm{TiO}_{2} / \mathrm{MEH}-\mathrm{PPV}$ polymer photovoltaics. Physical Review B 64(12): 125205-125214.

2. Becke A. D. (1988). Density-functional exchange-energy approximation with correct asymptotic behavior. Physical Review A 38(6): 3098-3100.

3. Nguyen-Cong H., Sene C. \& Chartier P. (1993). Photovoltaic cell based on cadmium sulfide sprayed film and electrolytic poly (3-methylthiophene): solvent nature effect. Solar Energy Materials and Solar Cells 30(2): 127-138

4. Kaeriyama K., Tanaka S., Sato M. \& Hamada K. (1989). Structure and properties of polythiophene derivatives. Synthetic Metals 28(1-2): 611-620.

5. Kuhn H. (1949). A quantum-mechanical theory of light absorption of organic dyes and similar compounds. Journal of Chemical Physics 17(12): 1198.

6. Lee C., Yang W. \& Parr R. G. (1988). Development of the Colle-Salvetti correlation-energy formula into a functional of the electron density. Physical Review $B$ 37(2): 785-789.

7. Mintmire J.W., White C.T. \& Elert M.L. (1988). Conformation and electronic structure of heterocyclic ring chain polymers. Synthetic Metals 25(2): 109-119.

8. Zotti G., Martina S., Wegner G. \& Schluter A.D. (1992). Well-defined pyrrole oligomers: electrochemical and UV/ vis studies. Advanced Materials 4(12): 798-801. 